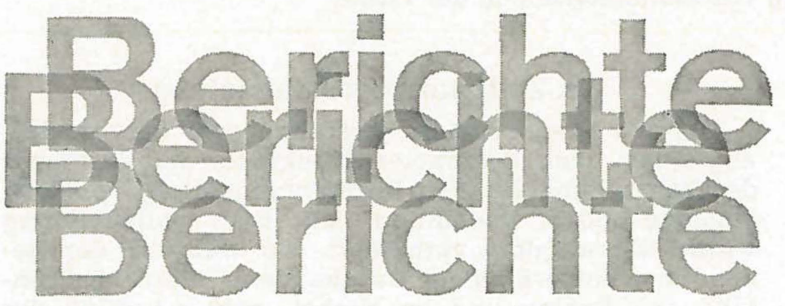

Anz. Schädlingskde., Pflanzenschutz, Umweltschutz 48, 113-118 (1975)

(C) 1975, Verlag Paul Parey, Berlin und Hamburg

ISSN 0003-6307/ASTM-Coden: ASPUCR

\title{
Zur Bekämpfung von Gallenläusen aus der Gattung Sacchiphantes [Adelgidae, Homoptera] an der Fichte [Picea abies (L.) Karsten]
}

\author{
Von J. K. MaKsYMOV
}

Mit 3 Abbildungen

\begin{abstract}
Contribution to the control of spruce gall aphids of the genus Sacchiphantes on Norway spruce
\end{abstract}

Active ingredients of $0,03 \%$ Lindane or $0,07 \%$ Endosulfan (spray) as well as of $6 \%$ Lindane or Endosulfan (fog) were entirely successful in the control of fundatrix-larvae. Spraying with 0,05\% Dioxacarb showed also a good action.

A description of the material and method used together with an outline of control-experiments abroad are given.

\section{Problemstellung}

Gallenläuse aus der Familie Adelgidae und den Gattungen Sacchiphantes und Adelges sind eine häufige Erscheinung an jungen Fichten und Lärchen. Sie begleiten beide Holzarten von den Tieflagen bis an die obere Waldgrenze. Von praktischer Bedeutung werden die Gallenläuse erst bei Massenbefall. Der physiologische Schaden, den sie verursachen, äußert sich bei der Lärche in einer vorzeitigen Verfärbung und anschließendem Abfall von Nadeln. Bei der Fichte kommt es zur Bildung und Häufung der auffälligen, ananasartigen Gallen, die das Wachstum hemmen und ein Absterben von Zweigen oder Ästen zur Folge haben können (Abb. 1, 2). Solche Schädigungen wirken sich besonders gravierend in Aufforstungen in Gebirgslagen aus, die vorwiegend Schutzwaldcharakter haben und wo dem Pflanzenwachstum bereits durch extreme klimatische Bedingungen Grenzen gesetzt sind. Von Bedeutung sind die Gallenläuse ferner in Christbaumkulturen, wo die Gallenbildung aus ästhetischen und kommerziellen Uberlegungen unerwünscht ist.

Es ist daher verständlich, daß bereits in der Vergangenheit Bekämpfungsversuche durchgeführt und Gegenmaßnahmen empfohlen wurden. Die vorliegende Versuchsserie erfolgte aus Anlaß neuer gesetzlicher Bestimmungen, die in der Schweiz die Verwendung von Lindan seit 1972 verbieten.

Ausnahmen sind auf wenige Fälle im Feld-, Garten und Obstbau beschränkt.

\section{Erfahrungen im Ausland}

2.1. Verwendete Insektizide

In der Fachliteratur der letzten 10 Jahre stammt etwa die Hälfte der Publikationen über die Bekämpfung von Adelgiden aus den Jahren 1972 und 1973. Die

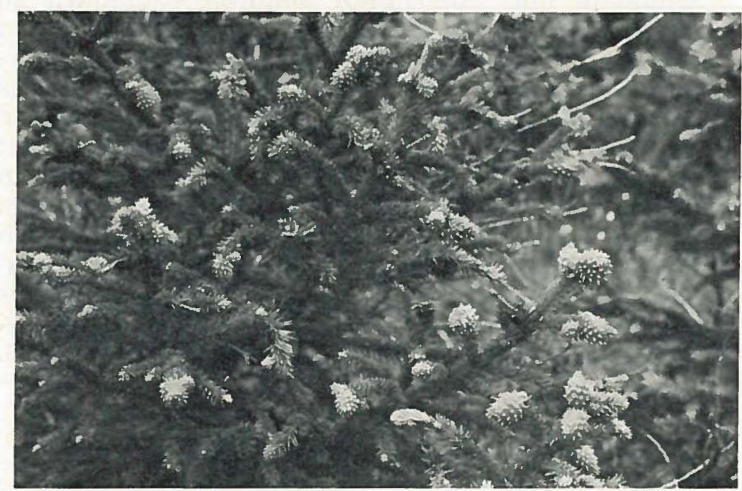

Abb. 1. Sacchiphantes-Gallen in einer Fichten-Aufforstung ob Andermatt, $1700 \mathrm{~m}$ ü. $\mathrm{M}$.

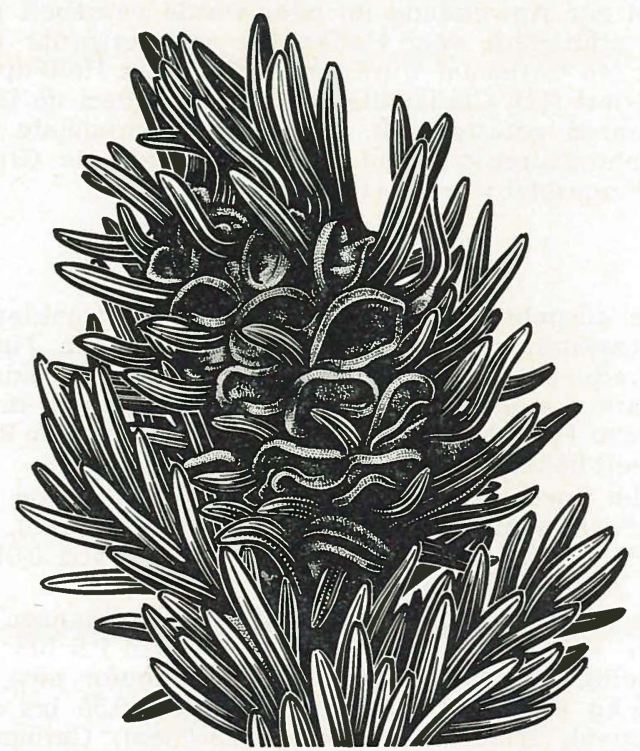

Abb. 2. Sacchiphantes-Galle, von Geflügelten verlassen (Zeichnung M. Šebek) 
für die Experimente verwendeten Insektizide basieren auf einer der folgenden vier Wirkstoffgruppen: Lindan, Phosphorsäureester, Endosulfan und Carbamate.

Mit einer Lindan-Nebellösung sowie durch Vernebeln von unverdünntem Mineralöl (Weißöl, Spindelölraffinat) haben wir bereits früher gute Resultate gegen Hiemosistens-Larven von Adelges laricis Vall. an der Lärche erzielt (9). Unsere positiven Erfahrungen mit dem erstgenannten Wirkstoff sind auch in anderen Versuchen in Europa $(1,2,6)$ und in den USA $(3,5)$ bestätigt worden. Lindan erwies sich ebenfalls gegen Adelgiden aus anderen Gattungen als wirksam: So gegen Dreyfusia an Abies alba Mill. (7), Gilletteella an Pseudotsuga menziesii (Mirb.) Fr. und Picea pungens Engelm. (11) sowie Pineus an Pinus patula Schlechtend. \& Cham. (12).

Am häufigsten wurden in Bekämpfungsversuchen Insektizide auf Phosphorsäureester-Basis eingesetzt. Ihre Wirkung war unterschiedlich, unabhängig davon ob es sich um systemische Mittel handelte oder nicht. Einerseits konnte die Gallenbildung an Fichten $(1,2$, $3,5,6,16)$ sowie der Befall durch Dreyfusia (7), respektive Gilletteella (13) wesentlich reduziert werden; anderseits war die Reduktion der Adelgiden sowohl an Fichten $(2,14,15,16)$ als auch an anderen Holzarten $(4,12)$ völlig ungenügend. Die letztere Erkenntnis deckt sich mit unseren Erfahrungen (9). Sie beruhen auf der Feststellung, daß im Zeitpunkt der Behandlung zwei Bedingungen erfüllt sein sollen, die einander häufig ausschließen: Relativ hohe Temperatur und keine Eiablagen.

Carbamate und Endosulfan gehören schließlich zu denjenigen Wirkstoffen, die häufig und mit Erfolg in Freilandversuchen gegen Adelgiden verwendet wurden. Die meisten Erfahrungen darüber stammen aus den USA. Ausnahmen bilden Bekämpfungsversuche gegen Adelgiden an der Fichte mit je einem Carbamat in Ungarn und Belgien $(6,15)$, mit Endosulfan in Dänemark (2) sowie gegen eine Pineusart mit beiden genannten Wirkstoffen in Kenya (12). Gegen Sacchiphantes abietis L. zeigten in den USA sowohl Präparate auf Endosulfan- (3) als auch auf Carbamat-Basis $(3,5,8)$ gute Wirkung. Beide erwähnten Wirkstoffe bewährten sich ferner gegen Gilletteella cooleyi Gill. $(4,11,13)$.

\subsection{Art der Behandlung}

Die Applikation aller obigen Wirkstoffe erfolgte in den meisten Fällen mittels eines tragbaren Rückensprühgerätes. Lindan kam vereinzelt auch als Stäubemittel zur Anwendung (6) oder wurde vernebelt $(7,9)$ oder schließlich vom Helikopter aus versprüht (11). Auch ein Carbamat wurde mit Hilfe eines Helikopters appliziert (11). Carbamate hat man außerdem im ULVVerfahren getestet (13). Systemische Carbamate und Phosphorsäureester sind schließlich auch als Granulate eingesetzt worden $(4,8,13,15)$.

\subsection{Wirkstoffaufwand}

Die ausgebrachten Wirkstoffmengen schwankten in den erwähnten Versuchen in weiten Grenzen. Neben Unterschieden in der Formulierung der verwendeten Präparate mag dabei auch die Heterogenität innerhalb der Phosphorsäureester und Carbamate eine Rolle gespielt haben.

Beim Sprühen variierte die Konzentration von 0,03 bis $2 \%$ (Lindan), von 0,05 bis $0,12 \%$ (Endosulfan), von $0,05 \%$ bis $2 \%$ (Phosphorsäureester) und von $0,01 \%$ bis $2 \%$ (Carbamate).

Bei Flächenbehandlungen erhielten die Pflanzen folgende Wirkstoffmengen pro 1 ha: Lindan 0,6 bis $1 \mathrm{~kg}$ (Nebeln), $1,13 \mathrm{~kg}$ (Sprühen vom Helikopter aus), 1,5 bis $2 \mathrm{~kg}$ (Stäuben). Phosphorsäureester 0,56 bis $2 \mathrm{~kg}$ (Sprühen). Endosulfan 0,56 kg (Sprühen). Carbamate 0,56 bis $1,69 \mathrm{~kg}$ (Sprühen), 1,13 kg (Sprühen vom Helikopter aus), 5,6 kg (Streuen, Granulat).

\subsection{Zeitpunkt der Behandlung}

Aus den besprochenen Versuchen geht hervor, daß, abgesehen vom geeigneten Insektizid, der Zeitpunkt der Behandlung für den Bekämpfungserfolg von ausschlaggebender Bedeutung ist. Die Gallenbildung wurde überall dort verhindert, wo während der Behandlung keine Eiablagen vorhanden waren: Im Frühjahr, vor Beginn und im Herbst, nach Abschluß der Vegetationszeit.

\section{Eigene Untersuchungen}

\subsection{Standorte und Insektizide}

Die vorliegende Versuchsserie umfaßt Versuche an drei verschiedenen Standorten: in Copera, Kanton Tessin (November 1970), im Versuchsgarten unseres Institutes (März 1972) und in Bremgarten, Kanton Aargau (März 1973). In allen drei Fällen standen uns Fichtenkulturen mit 1,5 bis $2 \mathrm{~m}$ hohen Pflanzen zur Verfügung. Sie stockten in Copera an einem NW-exponierten Steilhang (650 $\mathrm{m}$ ü. M.), an den übrigen beiden Standorten hingegen in ebener Lage (560 und $370 \mathrm{~m}$ ü. M.). Die Fichten zeigten mehrjährigen SacchiphantesBefall und waren zudem von Fundatrix-Larven besiedelt.

Zur Anwendung kamen die Insektizide

Lindan

[Gamma-1,2,3,4,5,6-Hexachlorcyclohexan]

Endosulfan

$[1,2,3,4,7,7$-Hexachlorbicyclo- $(2,2,1)-2$-hepten-5,6-

bisoxymethylensulfit] und

Dioxacarb

[2-(1,3-Dioxalan-2-yl)-phenyl-N-methyl-

carbamat].

Lindan ist ein Fraß- und Atemgift, zum kleineren Teil auch noch ein Berührungsgift. Endosulfan wirkt ebenfalls als Fraß- und Kontaktgift, desgleichen das Carbamat Dioxacarb. Die Giftwirkung des letztgenannten Insektizides erfolgt, ähnlich wie bei den Phosphorsäureestern, über die Hemmung der Cholinesterase. Allen drei Wirkstoffen gemeinsam ist die Eigenschaft, lokal ins Gewebe einzudringen, wo sie von den saugenden Larven mit dem Pflanzensaft aufgenommen werden.

Wir haben folgende Konzentrationen und Applikationsverfahren geprüft:
Copera:
Lindan $6 \%$ nebeln
Versuchsanstalt:
Lindan $6 \%$ nebeln
Endosulfan $12 \%, 9 \%, 6 \%$ nebeln; 0,07\% sprühen
Bremgarten: Endosulfan $6 \%, 3 \%, 1,5 \%$ nebeln Dioxacarb $1 \%$ nebeln, $0,05 \%$ sprühen

Alle Prozentangaben beziehen sich auf den Wirkstoffgehalt.

Die Lindan-Nebellösung bezogen wir gebrauchsfertig ${ }^{1}$ ). Die Endosulfan-Nebellösung haben wir durch Verdünnen der im Handel erhältlichen Melophen-Emulsion (35\% Endosul-

1) Dr. R. Maag AG, Dielsdorf ZH 
fan) mit Mineralöl (Weißöl, Spindelölraffinat) selbst hergestellt. Ähnliches gilt für das Vernebeln von Dioxacarb. Das Handelspräparat Elocron (50\% Dioxacarb), ein Spritzpulver, konnten wir im Mineralöl allerdings nicht auflösen, sondern nur suspendieren.

Das Sprühen erfolgte mit den beiden erwähnten Handelspräparaten als wäßrige Emulsion (Endosulfan), respektive Suspension (Dioxacarb). Zum Nebeln verwendeten wir das bereits in einer früheren Publikation erwähnte, seither weiter verbesserte, tragbare Nebelgerät "Swingfog" (9), zum Sprühen eine Rückenspritze mit Kolbenpumpe, Marke "Birchmeier Senior".

Die Behandlungen richteten sich gegen die an der Basis von Knospen befindlichen FundatrixLarven. Der Zeitpunkt wurde so gewählt, daß keine Eiablagen vorhanden waren und die Larven ihre Saugtätigkeit bereits aufgenommen (Frühjahr) oder noch nicht abgeschlossen haben (Herbst). Im Gegensatz zu Hiemosistens-Larven, die den Winter an der Lärche verbringen, scheiden Fundatrix-Larven an der Fichte dorsal röhrenförmige Wachsfäden aus (Abb. 3). Wenn gegen solche Larven mit reinem Mineralöl (Weißöl, Spindelölraffinat) vorgegangen wird, verhindern die Wachsfäden die Bildung eines lückenlosen Olfilms, der eine Erstickung der Larven zur Folge hätte. Darum ist eine Bekämpfung von Gallenläusen an der Fichte mit reinem Mineralöl, ohne anderen Wirkstoff, unbefriedigend, an der Lärche hingegen erfolgreich (9).

\subsection{Versuchsplan}

Mit Ausnahme von Copera, wo eine Flächenbehandlung mit nur einem Insektizid vorgesehen war, haben wir eine ähnliche Versuchsanordnung vorgenommen, die wir bereits beschrieben haben (9). Pro Versuch wurden 54 Fichten mit Sacchiphantes-Befall ausgewählt, mittels Aluminiumfolien fortlaufend numeriert sowie mit Hilfe von Zufallszahlen den fünf verschiedenen Behandlungen und der unbehandelten Kontrolle zugeordnet.

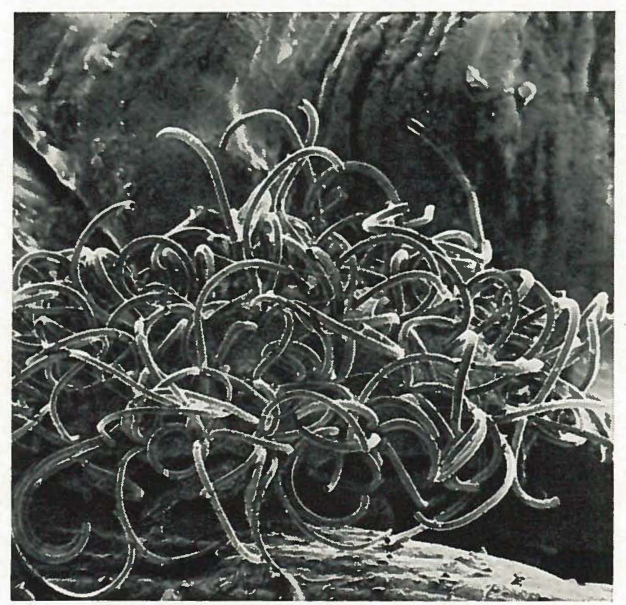

Abb. 3. Uberwinternde Fundatrix-Larve aus der Gattung Saca Dorsale Wachsfäden normal ausgebildet $(120 \mathrm{x})$
Zu jeder Behandlungsart gehörten somit neun Pflanzen, die als Wiederholungen bewertet wurden. Während der Applikation eines Insektizides waren alle übrigen Versuchsbäume, die eine andere Zuordnung hatten, mit Plastiksäcken zugedeckt.

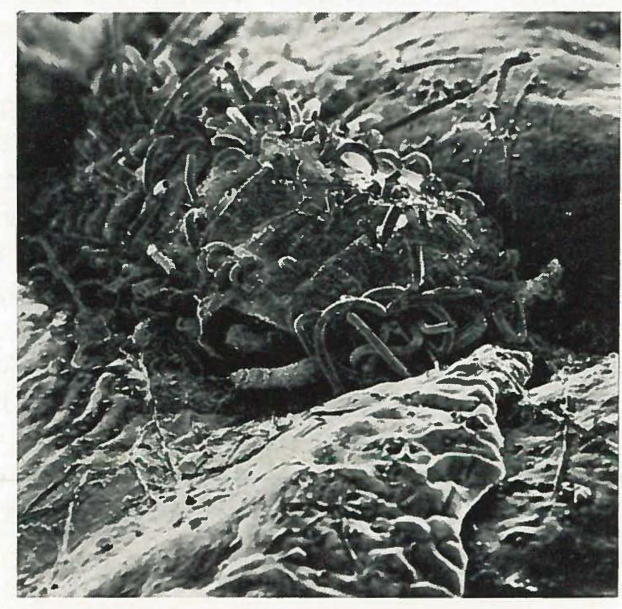

b Dorsale Wachsfäden teilweise entfernt (136 x)

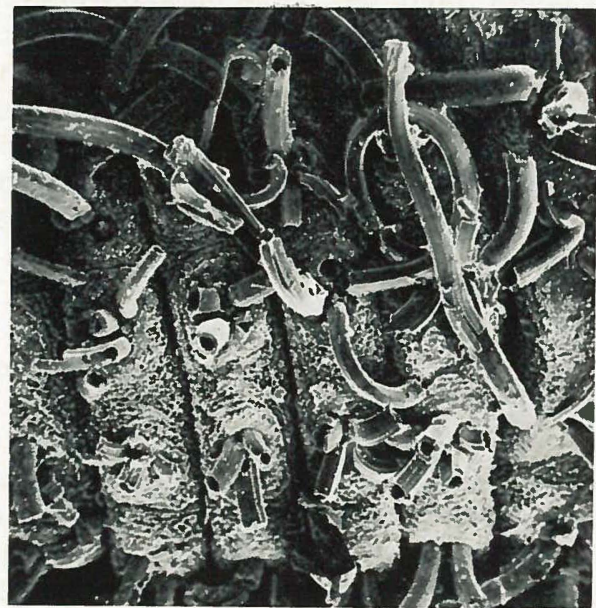

c Dorsale Segmente mit abgebrochenen Wachsfäden (348 x)

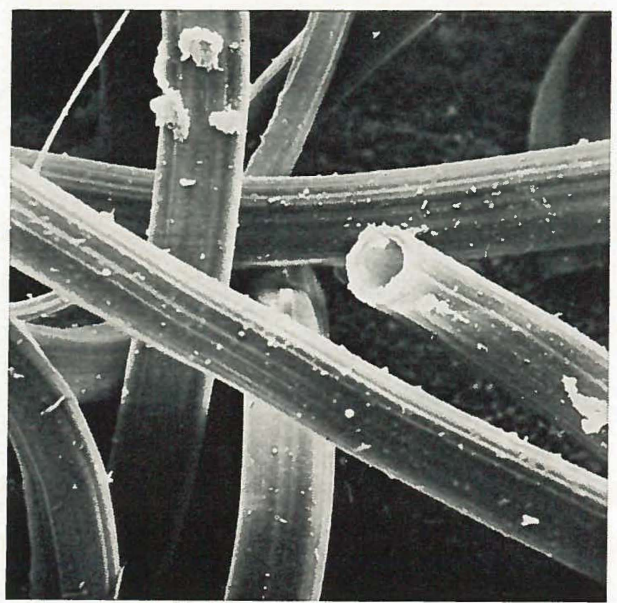

d Rohrartige Wachsfäden (1136 x) (Rasterelektronenmikroskop, Aufnahmen P. Wägli, Lab. für Elektronenmikroskopie II, ETH Zürich) 
Den Wirkungsgrad der Behandlung haben wir durch Auszählen von lebenden und toten Larven an behandelten sowie an unbehandelten Fichten geprüft. Pro Baum wurden je nach Befallsstärke mindestens vier Zweige zufällig entnommen und an jedem Zweig höchstens zehn Larven fortlaufend ausgezählt. Die Gesamtzahl kontrollierter Larven pro Versuchsbaum betrug 40. Die Kontrolle erfolgte im Labor am Stereomikroskop bei 25- bis 50facher Vergrößerung.

In Abweichung von den obigen Angaben haben wir bei der Flächenbehandlung in Copera keine unbehandelten Fichten ausgeschieden. Als Kontrollbäume dienten je 10 zufällig ausgewählte Pflanzen vor und nach der Behandlung. Da diese Pflanzen starken Gallenlausbefall zeigten, beschränkten wir die Probeentnahme auf drei Zweige pro Baum. Vor der Behandlung wurden alle Larven, nach der Behandlung hingegen maximal 50 Larven pro Zweig ausgezählt.

Die Erfolgskontrolle geschah außerdem durch Auszählen von Gallen an 100 Fichten vor und nach der Behandlung.

\subsection{Versuchsdurchführung}

In Copera fand der Bekämpfungsversuch, im Gegensatz zu den beiden anderen Standorten, im Herbst statt (4.11.). Dieser Zeitpunkt hat, im Vergleich zum Frühjahr, oft den Vorteil stabilerer Wetterlage. Die Applikation des Insektizides kann in Angriff genommen werden, sobald alle Fundatrix- respektive Pseudofundatrix-Larven geschlüpft sind. Das ist in der Regel Ende September oder im Oktober der Fall.

Unsere Behandlung umfaßte 16 Aren und wurde mit Hilfe des "Swingfog" vorgenommen. Sie erfolgte am späten Nachmittag bei einer Temperatur von rund $10^{\circ} \mathrm{C}$ unter Ausnutzung eines schwachen Bergwindes. Dieser drückte den Lindannebel vom oberen Rand der Pflanzung durch die ganze Fläche über eine Distanz von $40 \mathrm{~m}$ hindurch. Der Geräteführer hatte nur den Horizontalweg oberhalb der Pflanzung langsam ab- zuschreiten. Es wurde darauf geachtet, daß der Nebel etwa eine Minute lang am gleichen Ort einwirken konnte. Die Behandlung dauerte bei Verwendung einer Wirkstoffdüse von $0,9 \mathrm{~mm}$ ungefähr eine halbe Stunde. Es wurden rund 5 Liter Nebellösung verbraucht.

Im Garten der Versuchsanstalt und in Bremgarten wurden die Bekämpfungsversuche im Frühjahr durchgeführt (24. 3. 72, 28. 3. 73). Sie fanden unter vergleichbaren klimatischen Bedingungen statt. Die Temperatur lag 1972 bei $14^{\circ}$ bis $15{ }^{\circ} \mathrm{C}$ und 1973 bei $15^{\circ} \mathrm{C}$ bis $16{ }^{\circ} \mathrm{C}$. Beidemal wehte eine flaue Brise (Windstärke 2 der Beaufort-Skala), die durch häufige Windböen gekennzeichnet war. Die stärkere Brise im März 1972 $(2,4 \mathrm{bis} 3 \mathrm{~m} / \mathrm{sec})$ hatte längere Behandlungszeiten zur Folge als der schwächere Wind (2 bis $2,2 \mathrm{~m} /$ sec) im folgenden Jahr.

1972 beanspruchte das Nebeln der 9 Versuchsbäume 6 Minuten, im folgenden Jahr hingegen nur die Hälfte dieser Zeit. Für das Sprühen mußte in beiden Jahren gleichviel oder mehr Zeit aufgewendet werden als für das Nebeln (6, respektive 4,5 Minuten). Der Verbrauch an Nebellösung betrug 1972, bei Verwendung einer Düse von $0,9 \mathrm{~mm}$, ca. 1 Liter für 9 Versuchsbäume; ein Jahr später, bei schwächerem Wind und der dadurch bedingten kürzeren Behandlungsdauer, etwa 0,5 Liter. Die letztgenannte Insektizidmenge wurde beim Sprühen (Düse 0,75 mm $\phi$ ) für jeden der Versuchsbäume verbraucht.

\subsection{Resultate}

In Ubereinstimmung mit Erfahrungen im Ausland geht aus den Ergebnissen unserer Versuche hervor, daß die Bekämpfung von Gallenläusen an der Fichte außer im Frühjahr auch im Herbst möglich ist. Wie aus der Tabelle 1 ersichtlich ist waren am 26. November, 22 Tage nach der $\mathrm{Be}$ handlung mit $6 \%$ Lindannebel, von den rund 1000 ausgezählten Larven an 10 Versuchsbäumen keine am Leben.

Tabelle 1

Versuchsresultate Copera

\begin{tabular}{|c|c|c|c|c|c|c|}
\hline \multicolumn{4}{|c|}{ Behandlung: 4.11.1970 } & \multicolumn{3}{|c|}{ Erfolgskontrolle: 26.11. 1970} \\
\hline \multicolumn{4}{|c|}{ Unbehandelt } & \multicolumn{3}{|c|}{ Lindan - Nebellösung $6 \%$} \\
\hline \multirow{3}{*}{$\begin{array}{l}\text { Baum } \\
\text { Nr. }\end{array}$} & \multirow{2}{*}{\multicolumn{2}{|c|}{$\begin{array}{l}\text { L a r v e n } \\
\text { A n z a h l }\end{array}$}} & & Baum & Larven & \\
\hline & & & & Nr. & Anzahl & \\
\hline & lebend & tot & lebend & & lebend & tot \\
\hline 26 & 14 & 46 & 23,3 & 23 & 0 & 150 \\
\hline 222 & 130 & 66 & 66,3 & 191 & 0 & 55 \\
\hline 235 & 289 & 343 & 45,7 & 234 & 0 & 92 \\
\hline 236 & 49 & 8 & 86 & 235 & 0 & 125 \\
\hline 241 & 37 & 10 & 78,7 & 242 & 0 & 104 \\
\hline 246 & 217 & 97 & 69,1 & 245 & 0 & 74 \\
\hline 278 & 72 & 53 & 57,6 & 279 & 0 & 71 \\
\hline 456 & 172 & 83 & 67,5 & 455 & 0 & 50 \\
\hline 514 & 127 & 27 & 82,5 & 513 & 0 & 150 \\
\hline 647 & 123 & 32 & 79,4 & 646 & 0 & 117 \\
\hline Total & 1230 & 765 & 61,7 & Total & 0 & 988 \\
\hline
\end{tabular}


Die zur gleichen Zeit an Zweigen von 10 anderen Fichten ermittelten Lindanrückstände schwankten zwischen 0,74 und 1,76 ppm (Tab.2). Die zur Rückstandsanalyse verwendete Methode wurde bereits in anderem Zusammenhang beschrieben (10).

Tabelle 2

Versuchsresultate Copera, November 1970 Lindanrückstände in Zweigen und Nadeln junger Fichten, 22 Tage nach der Behandlung

\begin{tabular}{|c|c|c|c|}
\hline \multicolumn{3}{|c|}{ Zweige und Nadeln } & \multirow{3}{*}{$\begin{array}{l}\text { Lindan- } \\
\text { rückstände }\end{array}$} \\
\hline Fichte & Extrahiert & Aliquot & \\
\hline Nr. & & $\begin{array}{l}\text { gemessen } \\
\text { mg }\end{array}$ & \\
\hline 23 & 25 & 25 & 0,92 \\
\hline 191 & 20 & 20 & 1,46 \\
\hline 234 & 20 & 20 & 1,61 \\
\hline 242 & 25 & 25 & 1,46 \\
\hline 245 & 25 & 25 & 1,76 \\
\hline 279 & 25 & 25 & 1,19 \\
\hline 455 & 20 & 20 & 1,41 \\
\hline 513 & 25 & 25 & 1,00 \\
\hline 183 & 25 & 16 & 0,81 \\
\hline 493 & 20 & 20 & 0,74 \\
\hline
\end{tabular}

Erfassungsgrenze: 9,2 ng entsprechen 0,37 ppm $\left(1 \mathrm{ng}=10^{-9} \mathrm{~g}\right)$

Tabelle 3

Versuchsresultate EAFV 1972 Behandlung: 24. 3.; Erfolgskontrolle: 31. 5. Zahl überlebender Larven in Prozenten

\begin{tabular}{|c|c|c|c|c|c|c|}
\hline Behandlung & $\mathrm{U}$ & E3 & E2 & E1 & $\mathrm{L}$ & ES \\
\hline 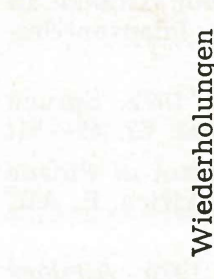 & 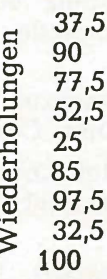 & $\begin{array}{l}0 \\
0 \\
0 \\
0 \\
0 \\
0 \\
0 \\
0 \\
0\end{array}$ & $\begin{array}{l}0 \\
0 \\
0 \\
0 \\
0 \\
0 \\
0 \\
0 \\
0\end{array}$ & $\begin{array}{l}0 \\
0 \\
0 \\
0 \\
0 \\
0 \\
0 \\
0 \\
0\end{array}$ & $\begin{array}{l}0 \\
0 \\
0 \\
0 \\
0 \\
0 \\
0 \\
0 \\
0\end{array}$ & $\begin{array}{l}0 \\
0 \\
0 \\
0 \\
0 \\
0 \\
0 \\
0 \\
0\end{array}$ \\
\hline Durchschnitt & 66,4 & 0 & 0 & 0 & 0 & 0 \\
\hline
\end{tabular}

$\mathrm{U}=$ unbehandelt

E1, E2, E3 = Nebeln mit Endosulfan $6 \%, 9 \%, 12 \%$ $\mathrm{L}=$ Nebeln mit Lindan $6 \% ; \mathrm{ES}=$ Sprühen mit Endosulfan $0,07 \%$

Der spätere Vergleich frisch gebildeter und vorjähriger Gallen hat ergeben, daß 705 Gallen an 100 Fichten vor der Behandlung nur deren vier an einer einzigen Pflanze nach der Behandlung gegenüberstanden.

Die Frühjahrsexperimente haben den Beweis erbracht, daß das Lindan vollwertig durch Endosulfan ersetzt werden kann, und daß auch Carbamate (Dioxacarb) für die Gallenlausbekämpfung geeignet sind (Tab. 3,4). Mit 6\% EndosulfanWirkstoff haben wir die beste Wirkung beim
Nebeln erzielt. Durch Herabsetzen der Konzentration wurde das Resultat spürbar verschlechtert (Tab. 4). Beim Sprühen zeigte eine 0,07\%ige Emulsion vollen Erfolg (Tab.3). Diese Konzentration könnte wahrscheinlich ohne Einbuße der Wirkung auf $0,05 \%$ herabgesetzt werden. Das Dioxacarb ist noch nicht derart erprobt wie das Endosulfan, zeigte jedoch in einer ersten Versuchsserie positive Resultate. Im Sprühverfahren wurde bei Verwendung einer 0,05\%igen Suspension eine gute Wirkung erzielt. Das Vernebeln einer $1 \%$ igen Suspension hatte jedoch eine unzureichende Mortatlität der Larven zur Folge (Tab. 4). Diese kann durch ein Heraufsetzen des verwendeten Wirkstoffanteils zweifellos erhöht werden.

\section{Folgerungen für die Praxis}

1. Die Bekämpfung von Gallenläusen an der Fichte richtet sich gegen die unterhalb der Knospen (vor allem Endknospen) befindlichen Larven ("Stammütter").

2. Der richtige Zeitpunkt der Behandlung ist für den Bekämpfungserfolg von entscheidender Bedeutung. Dieser Zeitpunkt liegt entweder im frühen Frühjahr oder im Herbst. Die Behandlung muß innerhalb einer Zeitspanne vorgenommen werden, in der die Larven ihre Saugtätigkeit bereits aufgenommen (Frühjahr) oder noch nicht vollständig abgeschlossen haben (Herbst). Es dürfen keine Eiablagen vorhanden sein.

Tabelle 4

Versuchsresultate Bremgarten 1973

Behandlung: 28. 3.; Erfolgskontrolle: 31.5.

Zahl überlebender Larven in Prozenten

\begin{tabular}{|c|c|c|c|c|c|c|}
\hline Behandlung & $\mathrm{U}$ & E1 & $E^{1 / 2}$ & $\mathrm{E}^{1 / 4}$ & $\mathrm{D}$ & DS \\
\hline & 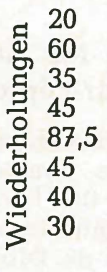 & $\begin{array}{l}0 \\
0 \\
0 \\
2,5 \\
0 \\
0 \\
0 \\
2,5 \\
7,5\end{array}$ & $\begin{array}{c}22,5 \\
17,5 \\
10 \\
27,5 \\
57,5 \\
0 \\
17,5 \\
0 \\
42,5\end{array}$ & $\begin{array}{l}12,5 \\
30 \\
50 \\
32,5 \\
37,5 \\
27,5 \\
7,5 \\
0 \\
47,5\end{array}$ & $\begin{array}{c}2,5 \\
7,5 \\
40 \\
35 \\
2,5 \\
2,5 \\
2,5 \\
0 \\
35\end{array}$ & $\begin{array}{l}0 \\
0 \\
0 \\
15 \\
7,5 \\
0 \\
0 \\
2,5 \\
0\end{array}$ \\
\hline Durchschnitt & 45,3 & 1,4 & 21,7 & 27,2 & 14,2 & 2,8 \\
\hline
\end{tabular}

$\mathrm{U}=$ unbehandelt $; E_{1}, E^{1 / 2}, E^{1 / 4}=$ Nebeln $\mathrm{m}$. Endosulsulfan $6 \%, 3 \%, 1,5 \%$

$\mathrm{D}=$ Nebeln mit Dioxacarb $1 \%$, DS $=$ Sprühen mit Dioxacarb $0,05 \%$

Die Unterschiede zwischen allen 6 Behandlungsarten sind nach dem verteilungsfreien Test von KRUSKAL und WALLIS signifikant

(2 P $<0,05)$. [Berechnung Dr. P. Schmid, EAFV.]

3. Für die Bekämpfung eignen sich besonders Präparate, deren Wirkstoffe lokal ins Gewebe eindringen und von den Larven mit dem Pflanzensaft aufgenommen werden. Die Präparate sollten auch bei Temperaturen unterhalb $10^{\circ} \mathrm{C}$ wirksam sein. 
4. Auf Grund unserer bisherigen Erfahrungen werden die unter 3. genannten Bedingungen am besten von Insektiziden auf Lindan- oder Endosulfan-Basis erfüllt. Wirkstoffanteile von $0,03 \%$ Lindan oder $0,07 \%$ Endosulfan beim Sprühen sowie von $6 \%$ Lindan oder Endosulfan beim Nebeln zeigten vollen Bekämpfungserfolg ${ }^{2}$ ).

5. Carbamate eignen sich ebenfalls für die Bekämpfung von Fichten-Gallenläusen. Da über ihren Einsatz unter unseren klimatischen Bedingungen noch zu wenig Erfahrungen vorliegen, sind weitere Untersuchungen erforderlich, um die optimalen Wirkstoffmengen zu ermitteln.

\section{Verdankungen}

Bei der Vorbereitung, Durchführung und Auswertung der Versuche haben mich zahlreiche Mitarbeiter tatkräftig unterstützt: Frau G. Geisel und Frau M. Simonett sowie die Herren Dr. R. G. Mitchell, Forsting. P. Brassel, E. Müller und W. Attinger. Ihnen allen möchte ich auch an dieser Stelle nochmals herzlich danken. Dank schulde ich ferner meinem Kollegen, Forsting. G. Beda, Stadtoberförster von Bremgarten, für die Uberlassung von Versuchsflächen. Die Insektizide Melophen und Elocron wurden uns in verdankenswerter Weise von den Firmen Dr. R. Maag AG, Dielsdorf, und CIBA-GEIGY, Basel, unentgeltlich zur Verfügung gestellt.

\section{Zusammenfassung}

Wirkstoffmengen von 0,03 Lindan oder $0,07 \%$ Endosulfan (Sprühen) sowie von 6\% Lindan oder Endosulfan (Nebeln) zeigten vollen Bekämpfungserfolg gegen Fundatrix-Larven. Gute Wirkung wurde auch mit 0,05\% Dioxacarb (Sprühen) erzielt.

Die Versuchsmethodik wird beschrieben sowie ein Uberblick über Bekämpfungsversuche im Ausland gegeben.

\section{Résumé}

Contribution à la lutte contre les Adelgides du genre Sacchiphantes sur l'épicéa

Les teneurs en matiere active suivantes ont été entièrement efficaces dans la lutte contre les larves fondatrices: $0,03 \%$ de Lindane ou $0,07 \%$ d'Endosulfan (aspersion) ainsi que $6 \%$ de Lindane ou d'Endosulfan (brouillard). L'aspersion de $0,05 \%$ de Dioxacarb accusait aussi une bonne action.

La publication donne également les indications sur le matériel et la méthode utilisés de même qu'un aperçu des essais sur la lutte à l'étranger.

\section{Sommario}

\section{Contributo alla lotta contro gli Afidoidei del genere Sacchiphantes sull'abete rosso}

I seguenti tenori di materie attive sono stati completamente efficaci nella lotta contro le larve fondatrici: Lo $0,03 \%$ di Lindano oppure lo $0,07 \%$ di Endosulfan (aspersione) nonchè il 6\% di Lindano oppure Endosulfan (nebulizzazione). Anche l'aspersione di Dioxacarb al $0,05 \%$ ha dato buoni risultati.

2) In der Schweiz kommt nur das Endosulfan in Frage, da die Verwendung von Lindan seit 1972 verboten ist.
Vengono inoltre descritti i metodi di prova ed i materiali utilizzati e sono indicate le esperienze fatte all'estero in questa lotta.

\section{Literaturverzeichnis}

1. Baurant, R., E. Seutin, A. Plasman, R. Caussin 1968: Le Parathion et le Lindane dans la lutte contre le Chermès de l'épicéa. Bull Rech. agron. Gembloux 3, 1, 3-14.

2. BeJER-Petersen, B., 1968: Nogle insecticider til bekaempelse af bladlusgaller (Sacchiphantes) på rødgran. [Some insecticides for the control of aphid galls (Sacchiphantes sp.) on Norway spruce (Picea abies)]. Dansk Skovforen. Tidsskr. 53, 105-112.

3. Cameron, E. A, R. L. Campbell, and L. E Adams, 1973: Insecticidal Control of Adelges abietis in Pennsylvania. J. Econ. Ent. 66, 3, 811-812.

4. Campbell, R. L., and C. P. Balderston, 1972: Insecticidal Control of Adelges cooleyi on Douglas-Fir in Ohio, with notes on Biology. J. Econ. Ent. 65, 3, $912-914$.

5. Campbell, R. L., and C. P. Balderston, 1972: Insecticidal Control of Eastern Spruce Gall Aphid During Autumn in Ohio. J. Econ. Ent. 65, 6, 17451746.

6. Gergácz, J., 1969: Chemische Bekämpfung der Sacchiphantes-Arten (Fam. Adelgidae) in Weihnachtsbaumkulturen. Erdész. Kut. 65, 1, 159-163.

7. Hochмuт, R., 1964: Problematika korovnice kavkazské (Dreyfusia nordmannianae Eckst.) v CSSR a ochrana proti ni. [Die Problematik der Tannentrieblaus (Dreyfusia nordmannianae Eckst.) in der ČSSR und ihre Bekämpfung]. Lesn. čas. 10 (37), 10, 879-902.

8. KERR, T. W., 1973: Carbofuran for Control of the Eastern Spruce Gall Aphid. J. Econ. Ent. 66, 3, 791.

9. MaKsymov, J. K., und P. Schmid, 1961: Die Bekämpfung der Fichtengallenlaus Adelges laricis Vall. Mitt. schweiz. Anst. forstl. Vers wes. 37, 5, 325-342.

10. Maksymov, J.K., 1974: Persistenz von Lindan an jungen Lärchen. Anz. Schädlingskde. Pflanzen-Umweltschutz 47, 113-115.

11. Nielsen, D. G., and C. P. Balderston, 1972: Spruce Gall Aphids. Ohio agr. Res. Dev. Cent. 62, 49-51.

12. Odera, J. A., 1972: Insecticidal Control of Pineus sp. (Homoptera: Adelgidae) in East Africa. E. Afr. agr. For. I. 34, 308-312.

13. Saunders, J. L., and D. A. Barstow, 1970: Adelges cooleyi (Homoptera: Phylloxeridae) Control on Douglas-Fir Christmas Trees. J. Econ. Ent. 63, 1, $150-151$.

14. Schindler, U., 1967: Zur Bekämpfung der Gallenläuse an Fichte. Forst-Holzwirt 22, 7, 135-136.

15. Seutin, E., et R. Baurant, 1971: De l'efficacité de quelques insecticides systémiques sur le Chermès de l'épicéa (Chermes tardus Dreyf.) Meded. Fac. Landbouww. Gent 36, 1, 266-273.

16. VAsIC, M., R. PERIN, 1973: Suzbijanje hermesa na smrči na Fruškoj Gori i Topčideru kod Beograda [Bekämpfung von Chermes an Fichte in Fruška Gora und Topčider bei Belgrad]. Šumarstvo 26, 9/10, $15-21$.

Anschrift des Verfassers: Dr. J.K. Maksymov, Eidgenöss. Anstalt f. d. forstliche Versuchswesen, $\mathrm{CH}$ 8903 Birmensdorf $\mathrm{ZH} /$ Schweiz. 